\title{
A Theoretical Study of Bulk Tungsten (W) Based on Momentum Transfer ( $q$-Dependent)
}

\author{
D. P. Rai and R. K. Thapa \\ Department of Physics, Mizoram University, Aizawl 796004, India \\ Correspondence should be addressed to D. P. Rai; dibyaprakashrai@gmail.com
}

Received 7 May 2014; Revised 28 July 2014; Accepted 25 August 2014; Published 8 October 2014

Academic Editor: Marco Consales

Copyright (C) 2014 D. P. Rai and R. K. Thapa. This is an open access article distributed under the Creative Commons Attribution License, which permits unrestricted use, distribution, and reproduction in any medium, provided the original work is properly cited.

\begin{abstract}
The ground state electronic properties of bulk (W) were studied within the density functional theory (DFT). We have also analyzed the momentum- $(q-)$ dependent loss function, dielectric constant, and optical conductivity (OC) within TD-DFT random-phase approximation (RPA). The loss function is plotted in the energy range $0-55 \mathrm{eV}$. The energy loss function spectrum shows four prominent peaks, two lower peaks below along with two sharp peaks above $30 \mathrm{eV}$. The different nature of peaks depends on the momentum transfer $q$. The peak caused by interband transition showed a less pronounced dispersion. From the dielectric function curve we have predicted the plasmon excitation at around $1.75 \mathrm{eV}$ and calculated the corresponding plasma frequency $\left(\omega_{p}\right)=$ $26.585 \times 10^{14} \mathrm{~s}^{-1}$.
\end{abstract}

\section{Introduction}

Tungsten (derived from the Swedish phrase "tung sten," meaning heavy stone) is a hard, brittle, steel-grey metal that is difficult to work with in the very pure state, unless it is malleable and ductile [1]. Tungsten is an interesting element whose unique physical properties make it an essential component in many industrial applications. Critical properties include very high melting point, very high density, hardness close to diamond, thermally stable, and excellent conductor [2]. The electronic structure and Fermi surface of tungsten have been studied extensively, experimentally and theoretically [3-7]. Some of the metallic crystals exhibit optical properties due to the interplay between electronic plasma screening and geometrical scattering effects [8]. Under suitable circumstances, this might be used to enhance thermal emission of photons in the visible region relative to lower frequencies and serve as a lighting technology [9]. Optical measurements provide several important microcharacteristics of the conduction electrons, their plasma frequency, and the relaxation frequency, as well as the energy gaps between the bands in the region of allowed electronic transitions. The optical properties of bulk polycrystalline tungsten have been investigated by Roberts [10] in the $0.5-0.8 \mu$ region and also by Lenham and Treherne [11] in the $0.35-20 \mu$ region. Juenker et al. measured the reflectivity of tungsten from 0.577 to $0.05 \mu$ [12]. Sudarshan et al. studied the angular distribution of backward reemitted positrons from a W(100) single crystal which showed that the mean energy of the emitted positrons increased with angular deviation from normal, with incident positron energies of 250 and $600 \mathrm{eV}$. The increase in the energy is explained in terms of energy-dependent refraction of positrons traversing the potential step at the surface [13]. Direct measurements of the electrical resistivity and the caloric equation of state for fluid tungsten have revealed that up to the pressure $7 \mathrm{GPa}$ the metal-nonmetal transition is the first-order phase transition, in which fluid tungsten coincides with the liquid-vapor transition [14]. The physical properties of solids, such as DOS, specific heats, the Debye temperature, neutron-diffraction spectra, heat conduction, and the electron-phonon interaction, are related to their lattice-dynamical behavior. Thus the dynamical behavior can be calculated by phonon dispersion in symmetry directions $[15,16]$.

Tungsten tips have become promising nanoprobes for scanning tunneling microscopy (STM) [17], nanoindentation and nanomanipulation in scanning electron microscopes 
(SEM) [18], and contact experiments inside a transmission electron microscope (TEM) [19]. In recent years the improvement of spatial resolution of atomic and subatomic particles as well as the nanoscale quantum wire, quantum dots, and quantum well is in tremendous progress with improving the capabilities of transmission electron microscope (TEM) [20, 21]. Electron energy loss spectrometry (EELS) in the TEM is a technique to study the loss function and the dielectric tensor $\varepsilon$ [22]. Since the information about optical and electronic properties is related to $\varepsilon$, thus the loss function is a key quantity. Other methods like inelastic X-ray scattering and multiple-scattering calculations of electron energy loss nearedge can also give information about the loss function [23]. Alkauskas et al. studied the $q$-dependent loss function of bulk Ag using a computational code "exciting" based on time dependent density functional theory (TD-DFT) which is well accurate in predicting the $q$-dependence of interband transitions [24].

\section{Computational Details}

The ground state density of states (DOS) and band structure of bulk cubic tungsten were calculated within generalized gradient approximation (GGA) [31] based on DFT [32] which employed wien $2 \mathrm{k}$ code [33]. Wave function was expanded up to cut-off parameter $R_{\mathrm{MT}} \times K_{\max }=10$, where $K_{\max }$ is the maximum value of the reciprocal lattice vector in the plane wave expansion and $R_{\mathrm{MT}}$ is the smallest atomic sphere radius of all atomic spheres. The calculations of optical and thermal properties were performed within the time dependent density functional theory (TD-DFT) based on a computational code called exciting-lithium [34]. The loss function is given by $\operatorname{lm}(-1 / \varepsilon)=u \operatorname{lm} \chi$. Here, $\chi$ is the density-density response function, $u$ is the Coulomb interaction, and $\varepsilon$ is the dielectric function. Within TD-DFT, the interacting response function $\chi$ has been obtained in the form of Dyson equation, $\chi=\chi_{o}+$ $\chi_{o}\left(u+f_{x c}\right) \chi[35]$, where $\chi_{o}$ is the Kohn-Sham independentparticle polarizability and $f_{x c}$ is the exchange-correlation kernel, the functional derivative of exchange-correlation potential with respect to electron density. By setting $f_{x c}$ to zero, exchange and correlation effects in the electron response are neglected and one obtains the random phase approximation (RPA). The calculation of $q$-dependent loss function was performed in two steps. In first step $q$-dependent loss function was calculated with local field effect (LFE) with single $q$ point and in the second step LFE has been neglected and loss function was obtained at different set of $q$ points. An off-symmetry $k$-point mesh has been taken to be $20 \times 20 \times 20$.

\section{Crystal Structure}

The X-ray diffraction (XRD) studies show that bulk tungsten crystallizes in cubic (bcc) structure having space group $\operatorname{Im} 3 \mathrm{~m}$ as shown in Figure 1. The molar volume and lattice constant are $9.546 \mathrm{~cm}^{3} /$ mole and $3.1738 \AA$, respectively [36].

\section{Results and Discussion}

The ground state $(T=0 \mathrm{~K})$ volume and bulk modulus were calculated from volume optimization method based on

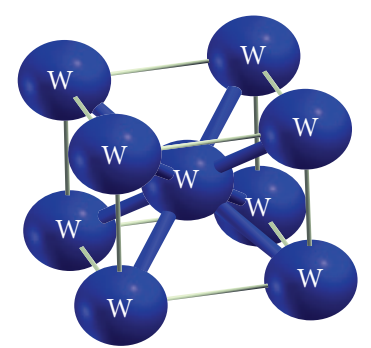

FIGURE 1: Crystal structure of tungsten (W).

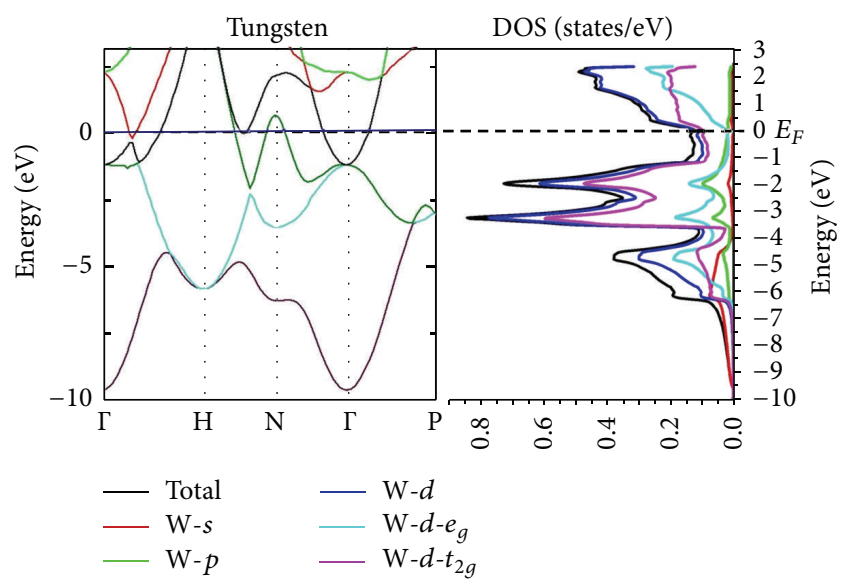

FIGURE 2: Electronic DOS and band structure.

Murnaghan's equation of states [37]. The FP-LAPW results reported here are found to be in agreement with the scalarrelativistic pseudopotential calculations of Bylander and Kleinman [4], the nonrelativistic pseudopotential calculation of Zunger and Cohen [27], and a calculation using an independently developed LAPW program by Jansen and Freeman [5]. The calculated volume and bulk modulus are compared with the experimental results, tabulated in Table 1.

The density of states (DOS) and band structure computed within the FPLAPW-DFT are shown in Figure 2. The energy band shows the dominant features of $5 d\left(d-e_{g}, d-t_{2 g}\right)$ states. The symmetry points $\Gamma-\mathrm{N}$ along $\Delta$ direction represents the $6 s$ and $6 p$-type states, respectively. The contribution of $d-t_{2 g}$ states is maximum at the valence band just below the Fermi energy $\left(E_{F}\right)$, whereas the conduction band is due to $d-e_{g}$ and $d-t_{2 g}$ states. The $5 d$ energy band at second $\Gamma$ point shows the splitting of two levels at around $-1.50 \mathrm{eV}$. The energy bands along $\mathrm{H}$ and $\mathrm{P}$ directions in the valence region is due $d-e_{g}$ and $d-t_{2 g}$, respectively. The three energy lines red, black, and cyan cross the Fermi level along the $\Gamma-\mathrm{H}$ symmetry point. This result is consistent with semirelativistic calculation of Ahuja et al. [3]. In Figure 2 the energy difference of two bands along $\mathrm{H}$ symmetry gives the band width $(\Delta d)=E\left(\mathrm{H}_{\text {upper }}\right)-$ $E\left(\mathrm{H}_{\text {lower }}\right)=4.2-(-5.3)=9.50 \mathrm{eV}$. Our result of band width is less than the previous results $(11.486 \mathrm{eV}) \mathrm{B} 3 \mathrm{LYP}$, $(10.898 \mathrm{eV})$ FPLAPW-LDA, and $(12.078 \mathrm{eV})$ SPR-KKR [3]. The $s$ - $d$ shift is the difference of $\mathrm{H}-\Gamma$ which is equal to $(-5.5)-(-9.20)=3.70 \mathrm{eV}$ which agrees well with 3.742 and $3.795 \mathrm{eV}$ calculated from SPR-KKR [3]. The DOS in 
TABLE 1

\begin{tabular}{|c|c|c|c|c|c|}
\hline \multicolumn{3}{|c|}{ Bulk modulus (Kbar) } & \multicolumn{3}{|c|}{ Lattice constant $(\AA)$} \\
\hline Calculated & Expt. $(0 \mathrm{~K} / 298 \mathrm{~K})$ & Previous $(0 \mathrm{~K} / 298 \mathrm{~K})$ & Calculated & Expt. & Previous \\
\hline \multirow[t]{5}{*}{3475.766} & $3140 / 3110[25]$ & $3070 / 3030[26] / 3450[27]$ & 3.126 & $3.165[25]$ & 3.172 (GGA) [25] \\
\hline & & $3200[28] / 2970[4] / 3450[5]$ & & & 3.126 (LDA) [25] \\
\hline & & & & & $3.162[4] / 3.149[5] /$ \\
\hline & & & & & $3.164[6] / 3.20[28]$ \\
\hline & & & & & $3.232[29] / 3.07[30]$ \\
\hline
\end{tabular}

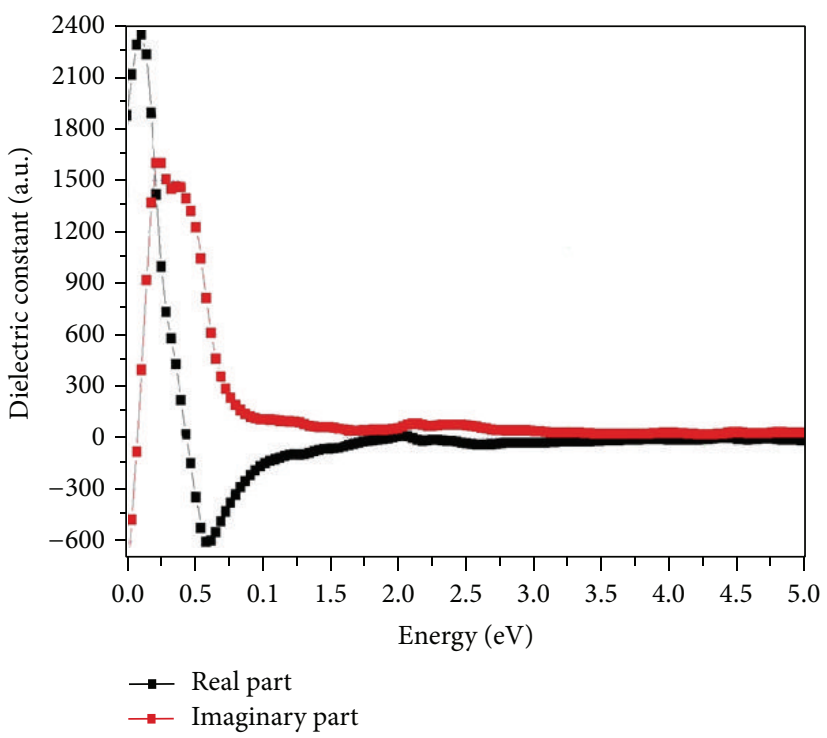

(a)

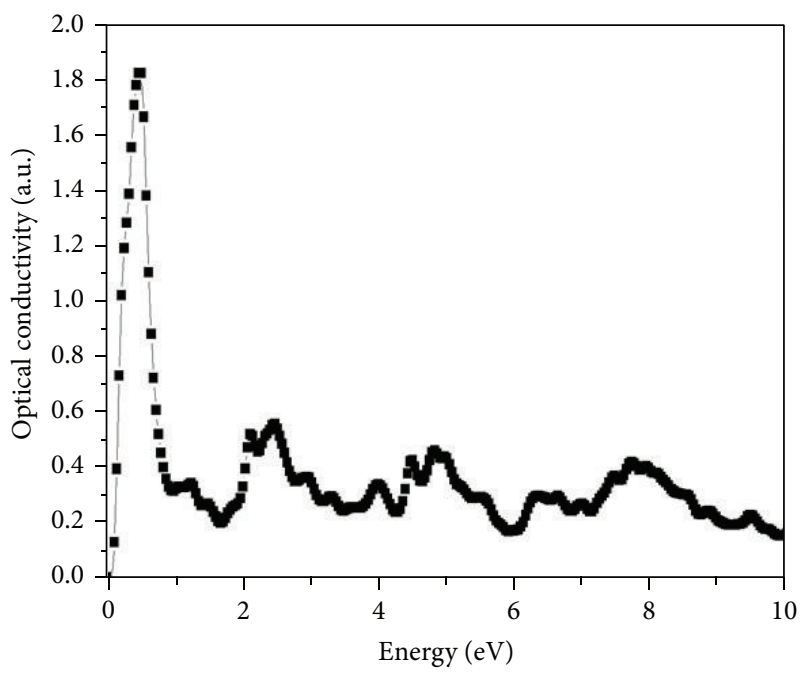

(b)

Figure 3: (a) Dielectric function and (b) optical conductivity.

Figure 2 shows the total and partial contributions of $6 s, 6 p$, and $5 d$ states. These curves along with the energy bandwidths confirm that valence band is a mixture of fully occupied $s, p$, and $d$ states. The band which crosses the Fermi level has small contribution compared to $d$ states and dominant contributions due to the $d$ states are prominent above the $E_{F}$.

4.1. Optical Properties. The interband dielectric function $(\varepsilon)$ and optical conductivity $(\sigma)$, together with the experimental trace $[38,39]$, are shown in Figures 3 and 4 . The experimental spectra show Drude behavior in the low energy region which is not found in theoretical results. The experimental static $\sigma$ at $0 \mathrm{eV}$ was $1250 \times 10^{14}$ esu [39]. A sharp rise in the imaginary part of dielectric constant $\left(\varepsilon_{2}\right)$ and the two close peaks at $0.3 \mathrm{eV}$ and $0.50 \mathrm{eV}$ has been observed which is consistent with the experimental maxima at $0.42 \mathrm{eV}$. These $\varepsilon_{2}$ peaks at around $0.3-0.5 \mathrm{eV}$ correspond to an interband transition along $(\mathrm{N}-\mathrm{N})$ and $(\Gamma-\Gamma)$ points (direct transition; see Figure 2). Above $0.50 \mathrm{eV} \varepsilon_{2}$ decreases and crosses zero at $1.75 \mathrm{eV}$ as there is no transition at high energies which contradicts the experimental results as additional maxima are evident at $1.82 \mathrm{eV}, 2.35 \mathrm{eV}, 3.42 \mathrm{eV}$, and $5.25 \mathrm{eV}$ [38]. The real part of dielectric function $\left(\varepsilon_{1}\right)$ increases up to $0.40 \mathrm{eV}$ and forms a peak which is in good agreement with the experimental results. With increasing photon energy the $\varepsilon_{1}$ decreases and crosses zero value at $0.52 \mathrm{eV}$ (negative slope) and $1.75 \mathrm{eV}$ (positive slope) shows a small deviation from the experimental results where the slopes are at $1.3 \mathrm{eV}$ and $5.2 \mathrm{eV}$ as shown in Figure 4(a). The zero crossing of $\varepsilon_{1}$ with positive slope at $1.75 \mathrm{eV}$ corresponds to volume plasmon $\hbar \omega_{P}$, where $\omega_{p}$ is the plasma frequency. The calculated plasma frequency is $26.585 \times 10^{14} \mathrm{~s}^{-1}$ underestimated by the experimental value $76 \times 10^{14} \mathrm{~s}^{-1}$ [39] and $91 \times 10^{14} \mathrm{~s}^{-1}$ from the tungsten gas [42]. The experimental optical conductivity is in close agreement with $\varepsilon_{2}$ (Figures 3(a), 4(a), and 4(b)). The high value of $\sigma$ indicates Drude behavior (below $1.0 \mathrm{eV}$ ) and decreases rapidly by virtue of participation in the conductivity of relaxing electrons [43], while in the energy region above $1.0 \mathrm{eV}$ the phenomena of electron transfer give a negligibly small contribution to $\sigma$. The similar curve can be seen in the infrared region illustrated in Figure 3(b). The experimental refractive index and extinction coefficient are shown in Figure 6(a). The increase in extinction coefficient as a function of wave length is similar to decrease in $\varepsilon_{2}$ and $\sigma$ with increasing energy.

Figure 5(a) represents the result of electron loss functions with and without LFE-RPA in the energy range $0-55 \mathrm{eV}$. The effect of LFE is found to be small; therefore, it can be neglected. The fact that this effect turned out to be negligible can be explained with four peaks which are in well 


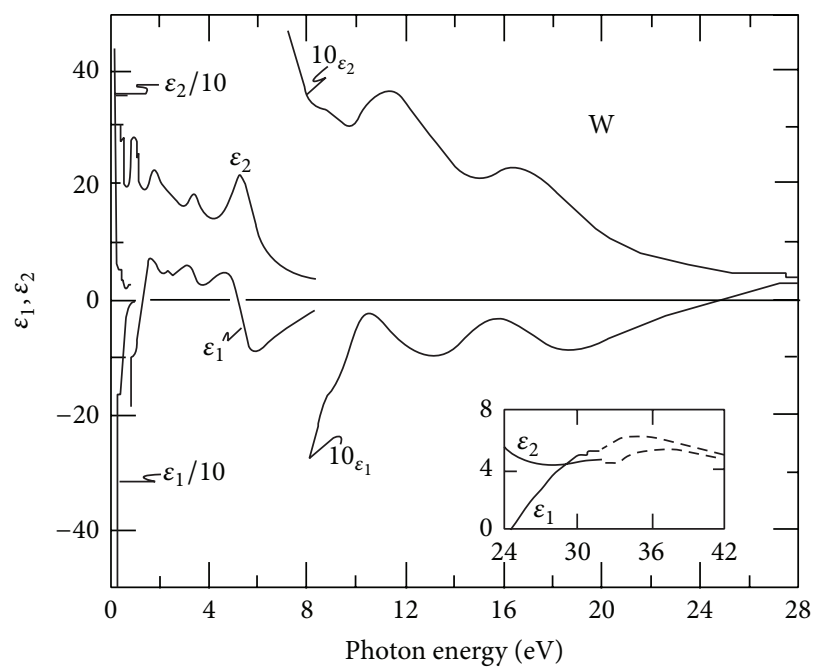

(a)

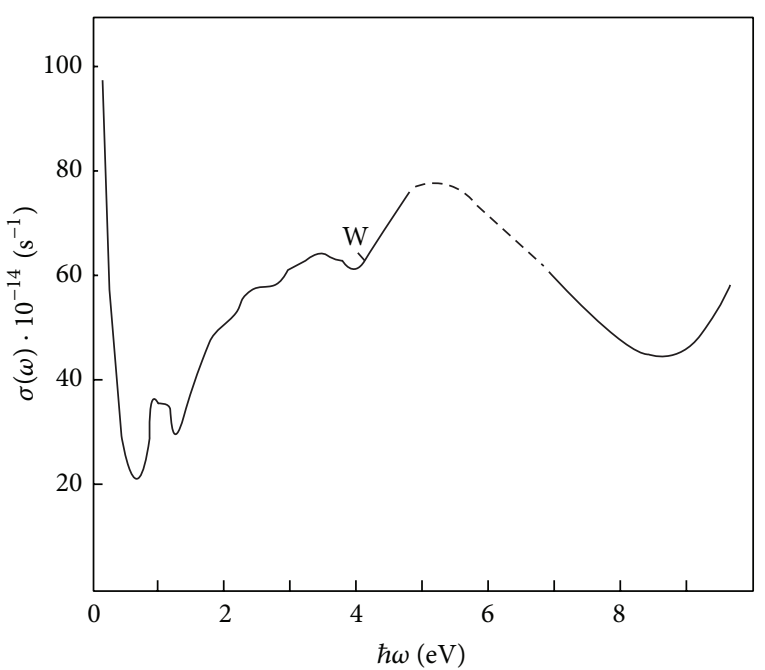

(b)

FIGURE 4: Experimental (a) dielectric function [38] and (b) optical conductivity [39].

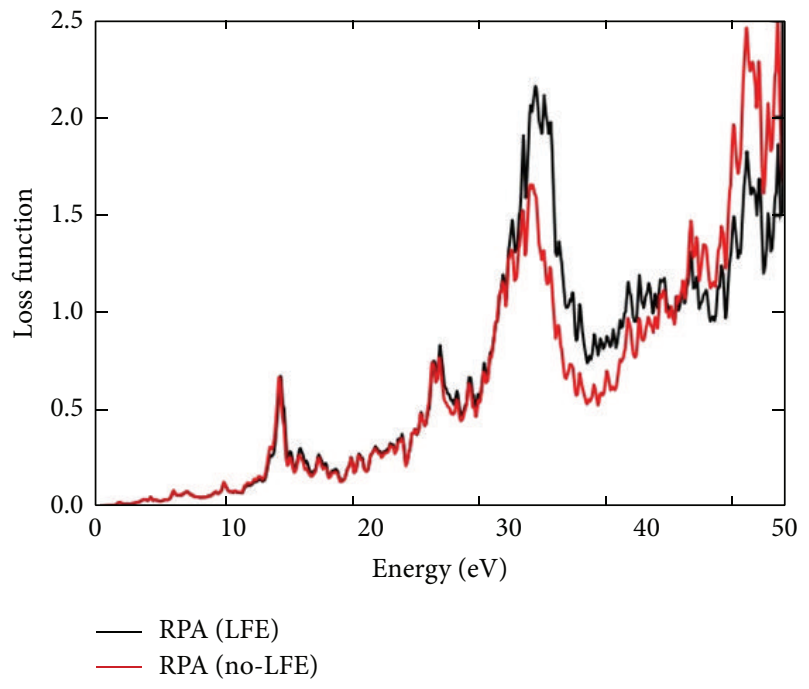

(a)

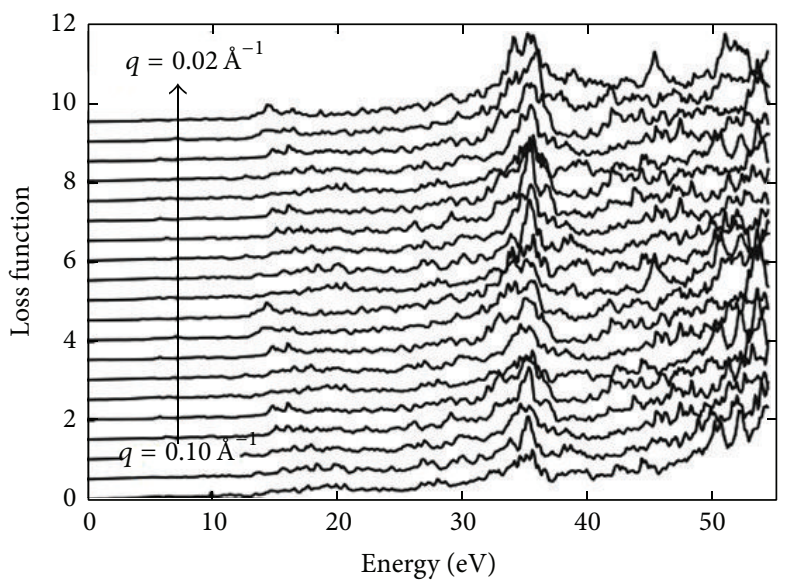

(b)

FIgURE 5: (a) Loss function (with and without local field) and (b) loss function at different $q$ points.

agreement with the experimental results. Our calculated peak positions are at $13 \mathrm{eV}, 28 \mathrm{eV}, 33 \mathrm{eV}$, and $51 \mathrm{eV}$ and agree well with the experimental peak positions at $10.0 \mathrm{eV}, 15.0 \mathrm{eV}$, and $25.3 \mathrm{eV}$ (Figures 5(a) and 6(b)). The maximum electron loss at $33 \mathrm{eV}$ underestimates the experimentally obtained maxima at $25.3 \mathrm{eV}$; this peak accounts interband activity near this range [38]. Similarly the result of other transition metals, obtained from simple free electron theory deviates largely as compared to experimental one [44]. As shown in Figure 5 the formation of volume plasmon corresponds to the first peak at $\sim 13 \mathrm{eV}$ which represents the collective excitation modes of all the valence electrons. This is also in close relation with the positive slope of vanishing $\varepsilon_{1}$ at $1.75 \mathrm{eV}$.
In experiment the stiffer slope of $\varepsilon_{1}$ indicates that the corresponding surface plasmon lies close to the volume plasmon, as shown in Figure 4(a) [38]. Figure 5(b) shows the loss function of bulk $\mathrm{W}$ without LFE for various momentum transfers $\left(q=0.02-0.10 \AA^{-1}\right)$, transfer vectors along [001] direction with broadening of $0.4 \mathrm{eV}$. The first two peaks appear at $20 \mathrm{eV}$ and $28 \mathrm{eV}$ almost flat and not well pronounced as shown in Figure 5(b). The width of the peak increases and the height decreases with $q$ which describes the quadratic dispersion as in the case of bulk Ag [24]. These results ascertain the plasmon is on the verge of damping. The peaks at around $\sim 33 \mathrm{eV}$ are broad and more prominent; the intensity of all the peaks decreases as a function of $q$. 


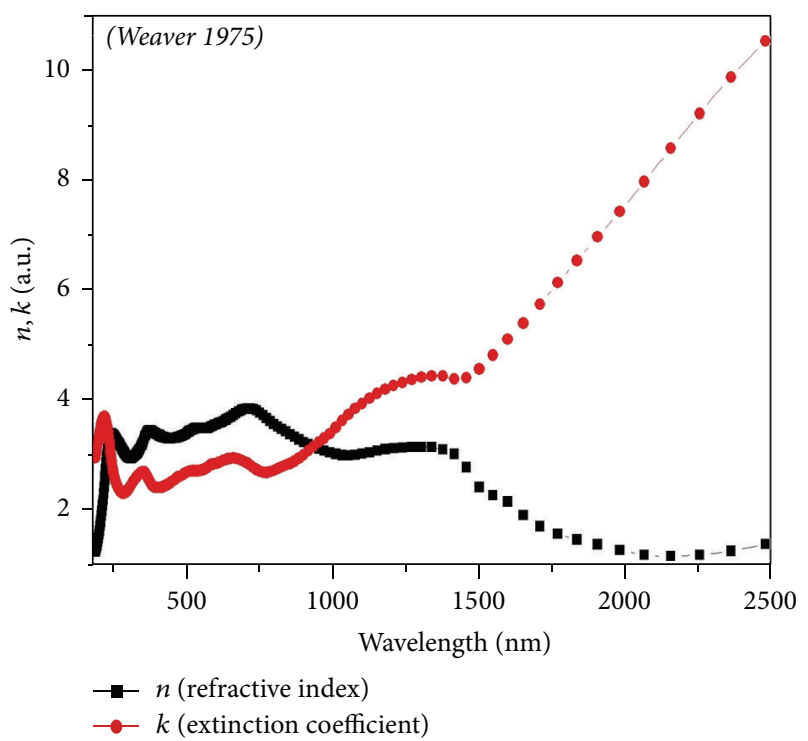

(a)

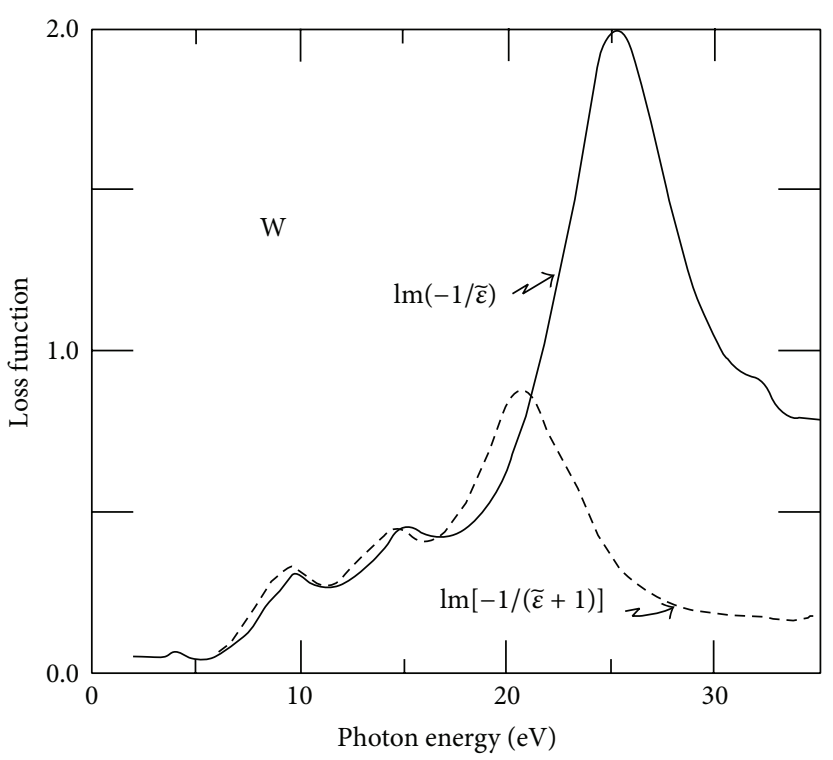

(b)

Figure 6: (a) Refractive index and extinction coefficient; (b) loss function: $\operatorname{lm}(-1 / \varepsilon)$ is the volume loss function and $\operatorname{lm}[-1 /(1+\varepsilon)]$ is the surface loss function [38].

At large $q$ the peaks almost become flat and the loss function approaches an almost continuum. The peaks at the lower energy below $30 \mathrm{eV}$ disappear much faster while the peaks at $35 \mathrm{eV}$ and $50 \mathrm{eV}$ are appreciable even for highest $q$ value.

4.2. Thermal Properties. In order to obtain the thermodynamic information about a system it is appropriate to begin with a complete description of the free energy of the system and its variation with temperature. This is best summarized by the free energy in temperature space as shown in Figure 7(b). This collection of data is useful as all the thermodynamic quantities are derived from the energy via the laws of thermodynamics [45]. This temperature relationship with other quantities provides a parameterization [46] which will be used according to classical thermodynamics to calculate the temperature dependence of several properties according to established equations such as the following:

$$
\begin{gathered}
S=-\frac{\partial F(T)}{\partial T}, \\
C_{p}=T \frac{\partial S}{\partial T}, \\
H=F(T)-T \frac{\partial F(T)}{\partial T},
\end{gathered}
$$

where $S$ is the entropy, $F$ is Helmholtz free energy, $H$ is enthalpy, $T$ is temperature, and $C_{p}$ is the heat capacity of a system.

Figure 7 demonstrates the temperature dependence of heat capacity, free energy, entropy, and internal energy up to $1500 \mathrm{~K}$. The calculated and experimental specific heat results agree well up to approximately $1500 \mathrm{~K}$ (Figure $7(\mathrm{a})$ ).
In experimental result at around $2500 \mathrm{~K}$ there is a sharp rise of $C_{p}$ (exponential growth above $2500 \mathrm{~K}$ ) (see [36]); this is not shown in our calculations. Ackland et al. have neglected both electronic and anharmonic corrections, thus giving them a less thorough yet conveniently more accurate volume expansion relationship [47]. There is no result of thermal expansion to compare with the experimentally determined thermodynamic and thermomechanical properties. The idea behind vibrational entropy is that a phase space is explored by atoms in a solid as they vibrate. The larger the phase space, the larger the vibrational entropy. As illustrated in Figure 7(c) at low energy there is a sudden rise in entropy. This is due to the fact that the atoms which are lined up give configurational entropy; another similar line would be disordered differently. For the stationary layers in the top, there is no absorption of heat when the temperature is increased, represented by a valley (Figure $7(\mathrm{c})$ ). On the other hand, the second lines will wiggle even more if the temperature is risen, so they will absorb heat. Thus the vibrational entropy will increase with temperature. In the following we use the conversion factor $1 \mathrm{Ha} \approx 2.194746 \times 10^{5} \mathrm{~cm}^{-1}$.

The phonon dispersion and phonon-DOS for symmetric and unstrained DOS are demonstrated in Figure 8. The $q$ points are given in reciprocal lattice coordinates for phonon calculation as given in exciting code [34]. By plotting the DOS on the same axes we are able to see the identical symmetric strains and the frequencies at which the peaks occur. The phonon dispersion within our investigation does not have flat transverse acoustic (TA) modes like in semiconductors [48]. The distinct features of optical branches are missing; acoustic band predominates. We have observed well-separated acoustic and optical branches only 


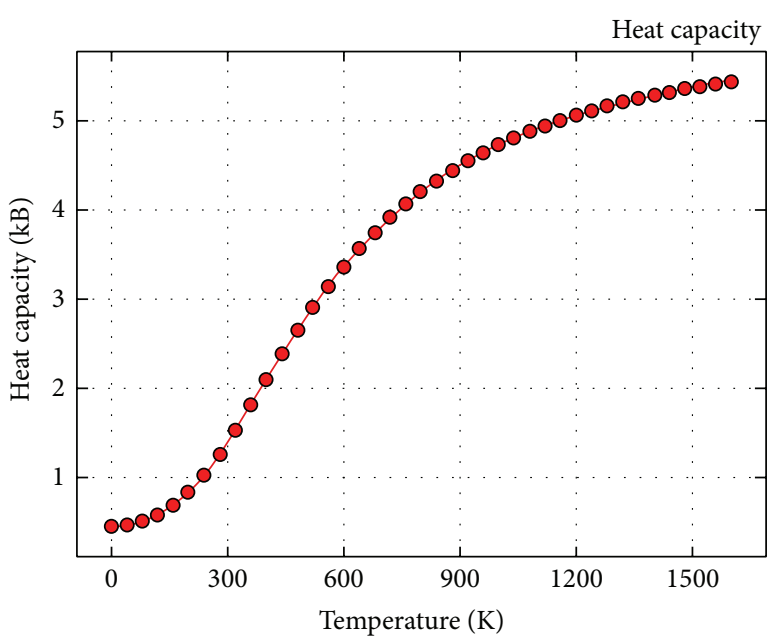

(a)

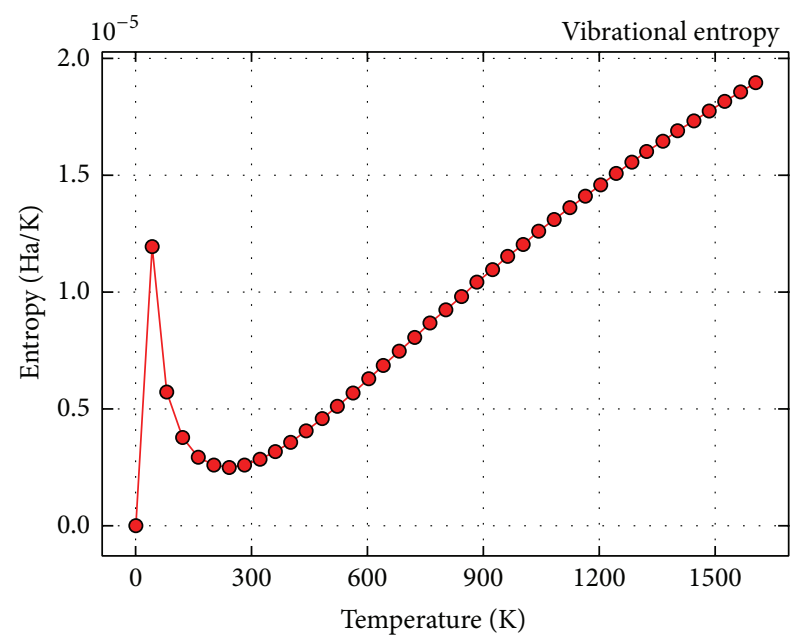

(c)

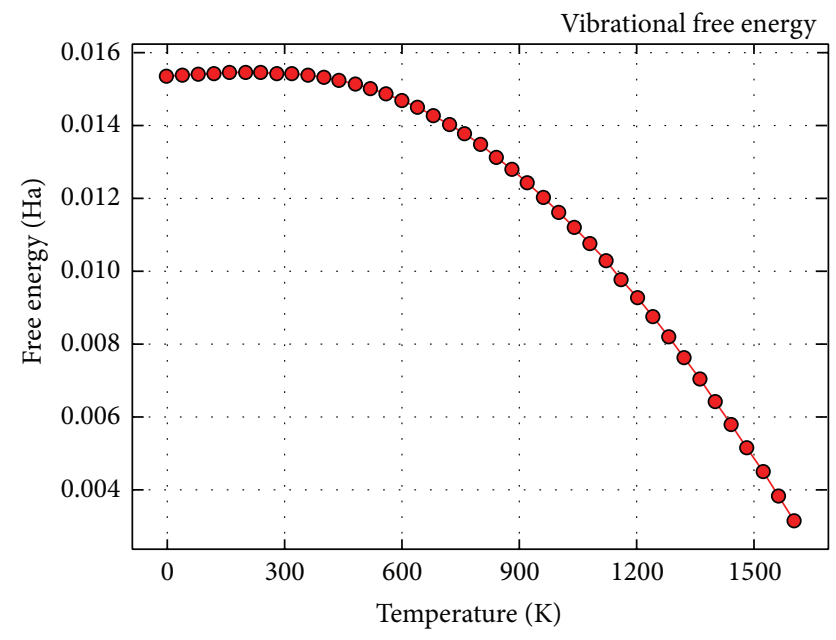

(b)

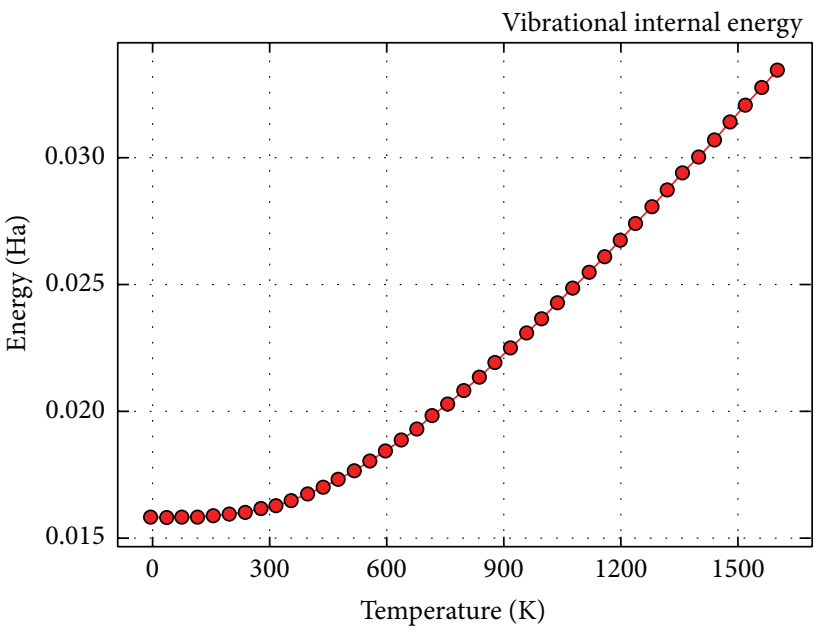

(d)

Figure 7: (a) Heat capacity, (b) free energy, (c) entropy, and (d) internal energy.

along X symmetry. The existence of overbending results in a sharp peak in the phonon-DOS above $\Gamma$ point similar to the case of second-order Raman spectrum [49]. Our results of phonon dispersion curve are consistent with the previous results calculated within embedded atom method (EAM) at $0 \mathrm{GPa}[40]$ and the experimental result calculated with thermal neutron inelastic scattering [41]. The pressure dependence of the phonon frequencies is due to the volume compression that comes from force constants, sensitive to external pressures. In all the calculated phonon-DOS, the two phonon peaks due to $q$ points in the reciprocal lattice coordinates are narrower as compared to previous result. Figure 9 illustrated the previous result of phonon-DOS; the broad peaks become narrower as lattice strain decreases from $4 \%$ to $0 \%$. A similar analysis was done by Ackland et al. [47], from which they infer that the impact of the phonon contributes to the free energy, since the calculation of free energy is related to an integration of the phonon-DOS. This is a very useful investigation because it allows neglecting computationally expensive lattice dynamics calculations. By simply calculating the ground state energy versus volume curve of a single primitive cell, the related elastic constants can be extracted.

\section{Conclusion}

In our present work we have studied the electronic structure of bulk tungsten by calculating DOS and band structure within DFT-GGA. The RPA TD-DFT often gives good results for loss spectra; however, it fails to give the description of absorption spectra of many bulk materials [50]. This is due to the screening potential associated with the bulk materials; in fact this is the result of electric dipole polarization which dampens the exchange-correlation effects. The loss function in case of TD-DFT (RPA) was studied within LFE and without LFE. The nature of loss function with LFE was 

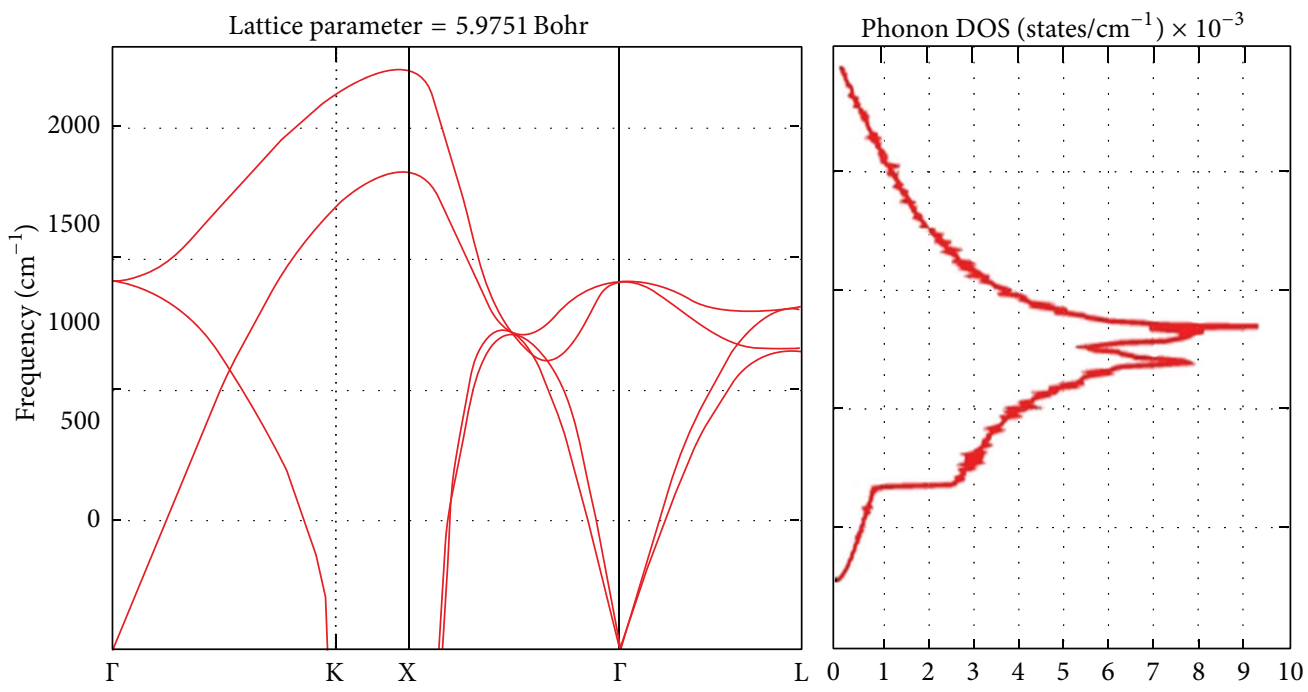

(a)

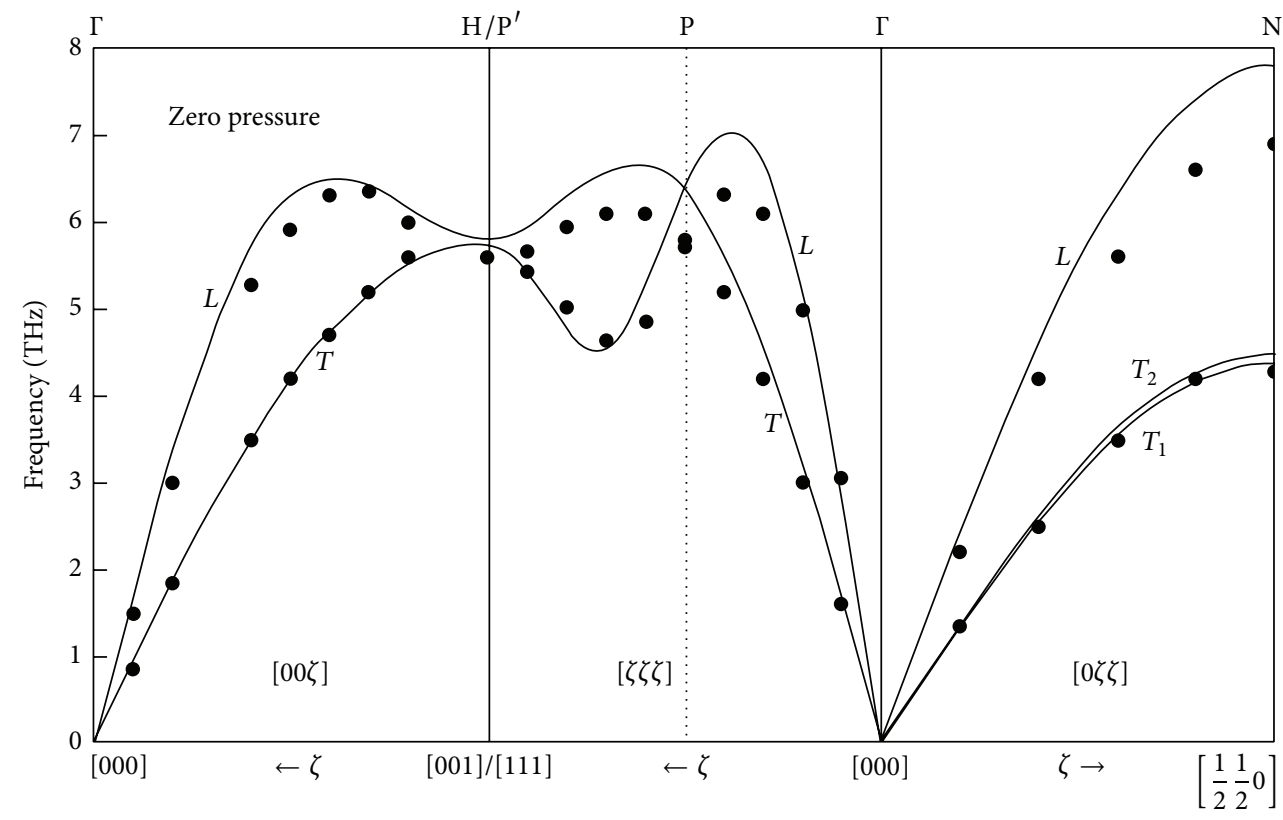

— MAEAM

- Exp.

(b)

Figure 8: (a) q-dependent phonon dispersion and phonon-DOS at 0\% strain; (b) phonon dispersion (solid line [40], expt. dotted line [41]).

similar with the loss function without LFE. The calculated loss function with a single $q$ point is in considerable agreement with the previous experimental result [38]. We have also performed the calculation of $q$-dependent loss function in the energy range $0-55 \mathrm{eV} ; q$ changes from 0.02 to $0.10 \AA^{-1}$ with broadening $0.40 \mathrm{eV}$. The electron energy loss peaks are $q$-dependent as the height of these peaks decreases with increasing $q$ which is attributed to damping of plasmon. We have shown the phonon dispersion relation of bulk $\mathrm{W}$ with $0 \%$ strain. The optical and acoustic branches are not well separated and distinct. The thermal properties showed metallic thermal behavior which agrees well with the previous results.

\section{Conflict of Interests}

The authors declare that there is no conflict of interests regarding the publication of this paper. 


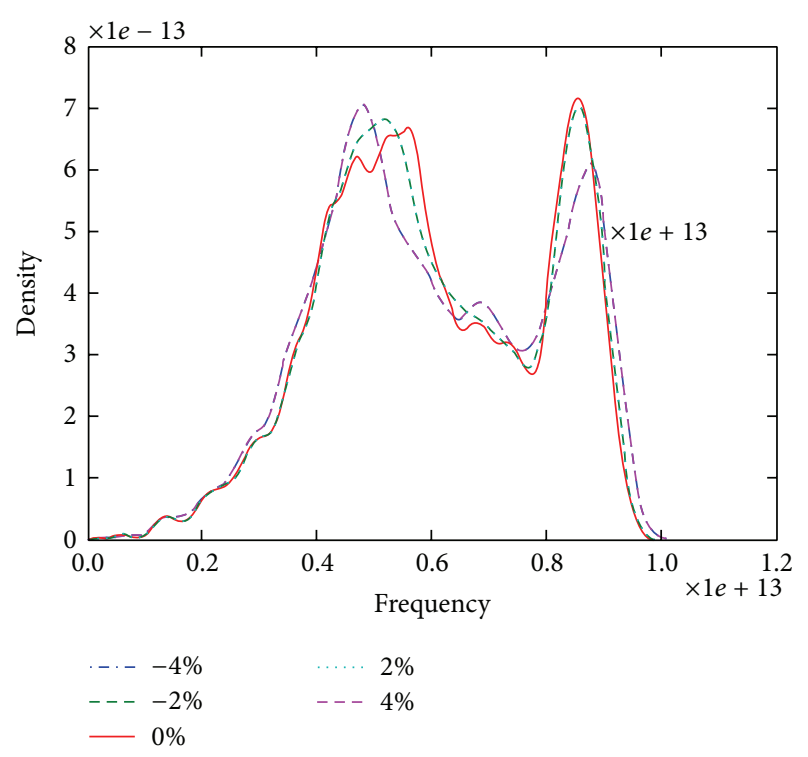

FIGURE 9: Calculated phonon-DOS at different strain lattice $(0 \%$, $\pm 2 \%$ and $\pm 4 \%$ ) [26].

\section{Acknowledgment}

D. P. Rai acknowledges Mr. Nichol Robert (nichol.18@ buckeyemail.osu.ed), Center for Electron Microscopy and Analysis (CEMAS), Ohio State University, Watts Hall, Columbus, $\mathrm{OH}$, for detailed information about this smart code, "exciting," providing related papers and fruitful suggestions.

\section{References}

[1] S. Albert, A Guide to the Elements, Oxford University Press, New York, NY, USA, 2nd edition, 2002.

[2] E. Paul DeGarmo, Materials and Processes in Manufacturing, MacMillan, New York, NY, USA, 5th edition, 1979.

[3] B. L. Ahuja, A. Rathor, V. Sharma, Y. Sharma, A. R. Jani, and B. Sharma, "Electronic structure and compton profiles of tungsten," Zeitschrift fur Naturforschung-Section A, vol. 63, no. 10-11, pp. 703-711, 2008.

[4] D. M. Bylander and L. Kleinman, "Self-consistent semirelativistic pseudopotential calculation of the energy bands, cohesive energy, and bulk modulus of W,' Physical Review B, vol. 27, no. 6, pp. 3152-3159, 1983.

[5] H. J. F. Jansen and A. J. Freeman, “Total-energy full-potential linearized augmented-plane-wave method for bulk solids: electronic and structural properties of tungsten," Physical Review B, vol. 30, no. 2, pp. 561-569, 1984.

[6] S.-H. Wei, H. Krakauer, and M. Weinert, "Linearized augmented-plane-wave calculation of the electronic structure and total energy of tungsten," Physical Review B, vol. 32, no. 12, pp. 7792-7797, 1985.

[7] A. Debernardi, M. Alouani, and H. Dreyssé, "Ab initio thermodynamics of metals: Al and W,' Physical Review B-Condensed Matter and Materials Physics, vol. 63, no. 6, Article ID 064305, 2001.
[8] A. Moroz, "Three-dimensional complete photonic-band-gap structures in the visible," Physical Review Letters, vol. 83, no. 25, pp. 5274-5277, 1999.

[9] Z.-Y. Li, "Modified thermal radiation in three-dimensional photonic crystals," Physical Review B, vol. 66, Article ID 241103R, 2002.

[10] S. Roberts, "Optical properties of nickel and tungsten and their interpretation according to drude's formula," Physical Review, vol. 114, no. 1, pp. 104-115, 1959.

[11] A. P. Lenham and D. M. Treherne, Optical Properties and Electronic Structure of Metals and Alloys, North Holland, Amsterdam, The Netherlands, 1966.

[12] D. Juenker W, J. L. LeBlanc, and R. C. Martin, "Optical properties of some transition metals," Journal of the Optical Society of America, vol. 58, no. 2, pp. 164-171, 1968.

[13] K. Sudarshan, S. N. Samarin, P. Guagliardo, V. N. Petrov, A. H. Weiss, and J. F. Williams, "Angle-resolved energy distribution of re-emitted positrons from a W(100) single crystal," Physical Review B: Condensed Matter and Materials Physics, vol. 87, no. 8, Article ID 085418, 2013.

[14] V. N. Korobenko and A. D. Rakhel, "Direct measurements of thermodynamic functions and electrical resistivity of fluid tungsten over a wide range of densities," Physical Review BCondensed Matter and Materials Physics, vol. 88, no. 13, Article ID 134203, 2013.

[15] B. Liu, M. Gu, L. X. Liu et al., "First-principles study of lattice dynamics and thermodynamics ofosmium under pressure," Chinese Physics B, vol. 19, no. 2, Article ID 26301, 2010.

[16] G. Wortmann, U. Ponkratz, B. Bielemeier, and K. Rupprecht, "Phonon density-of-states in bcc and hcp Eu metal under high pressure measured by ${ }^{151}$ Eu nuclear inelastic scattering of synchrotron radiation," High Pressure Research, vol. 28, no. 4, pp. 545-551, 2008.

[17] T. Kuzumaki, S. Kitakata, K. Enomoto, T. Yasuhara, N. Ohtake, and Y. Mitsuda, "Dynamic observation of the bending behavior of carbon nanotubes by nanoprobe manipulation in TEM," Carbon, vol. 42, no. 11, pp. 2343-2345, 2004.

[18] Y. Zhu, C. Ke, and H. D. Espinosa, "Experimental techniques for the mechanical characterization of one-dimensional nanostructures," Experimental Mechanics, vol. 47, no. 1, pp. 7-24, 2007.

[19] M. S. Bobji, J. B. Pethica, and B. J. Inkson, "Indentation mechanics of $\mathrm{Cu}-\mathrm{Be}$ quantified by an in situ transmission electron microscopy mechanical probe," Journal of Materials Research, vol. 20, no. 10, pp. 2726-2732, 2005.

[20] K. W. Urban, "Is science prepared for atomic-resolution electron microscopy?" Nature Materials, vol. 8, no. 4, pp. 260-262, 2009.

[21] M. Benaissa, W. Sigle, M. Korytov, J. Brault, P. Vennéguès, and P. A. van Aken, "Plasmon energy from strained GaN quantum wells," Applied Physics Letters, vol. 103, no. 2, Article ID 021901, 2013.

[22] R. F. Egerton, "Electron energy-loss spectroscopy in the TEM," Reports on Progress in Physics, vol. 72, no. 1, Article ID 016502, 2009.

[23] M. Wibbelt, H. Kohl, and P. Kohler-Redlich, "Multiplescattering calculations of electron-energy-loss near-edge structures of existing and predicted phases in the ternary system B-C-N," Physical Review B-Condensed Matter and Materials Physics, vol. 59, no. 18, pp. 11739-11745, 1999.

[24] A. Alkauskas, S. D. Schneider, S. Sagmeister, C. AmbroschDraxl, and C. Hébert, "Theoretical analysis of the momentumdependent loss function of bulk Ag," Ultramicroscopy, vol. 110, no. 8, pp. 1081-1086, 2010. 
[25] F. H. Featherston and J. R. Neighbours, "Elastic constants of tantalum, tungsten, and molybdenum," Physical Review, vol. 130, no. 4, pp. 1324-1333, 1963.

[26] E. Williams M, Ab-initio elastic and thermodynamic properties of high-temperature cubic intermetallics at finite temperatures [M.S. thesis], Brigham Young University, 2008.

[27] A. Zunger and M. L. Cohen, "Self-consistent pseudopotential calculation of the bulk properties of Mo and W," Physical Review $B$, vol. 19, no. 2, pp. 568-582, 1979.

[28] A. Debernardi, M. Alouani, and H. Dreyssé, "Ab initio thermodynamics of metals: $\mathrm{Al}$ and W," Physical Review B: Condensed Matter and Materials Physics, vol. 63, no. 6, Article ID 064305, 2001.

[29] P. W. Bridgman, "High Pressure Polymorphism," DaedalusJournal of the American Academy of Arts and Sciences, vol. 77, no. 1, p. $187,1949$.

[30] S. H. Chen and B. N. Brockhouse, "Lattice vibrations of tungsten," Solid State Communications, vol. 2, no. 3, pp. 73-77, 1964.

[31] J. P. Perdew, K. Burke, and M. Ernzerhof, "Generalized gradient approximation made simple," Physical Review Letters, vol. 77, no. 18, pp. 3865-3868, 1996.

[32] W. Kohn and L. J. Sham, "Self-consistent equations including exchange and correlation effects," Physical Review A, vol. 140, pp. A1133-A1138, 1965.

[33] P. Blaha, K. Schwarz, G. Madsen, D. Kvasnicka, and J. Luitz, An Augmented Plane Wave Plus Local Orbitals Program for Calculating Crystal Properties. Wien $2 K$ User's Guide, Technische Universität Wien, Vienna, Austria, 2008.

[34] J. K. Dewhurst, S. Sharma, and C. Ambrosch-Draxl, "Code development under the RT network EXCITING funded by the EU," contract HPRN-CT-2002-00317, 2005.

[35] M. Petersilka, U. J. Gossmann, and E. K. U. Gross, "Excitation energies from time-dependent density-functional theory," Physical Review Letters, vol. 76, no. 8, pp. 1212-1215, 1996.

[36] L. S. Dubrovinsky and S. K. Saxena, "Thermal expansion of periclase (MgO) and tungsten (W) to melting temperatures," Physics and Chemistry of Minerals, vol. 24, no. 8, pp. 547-550, 1997.

[37] F. D. Murnaghan, "The compressibility of media under extreme pressures," Proceedings of the National Academy of Sciences of the United States of America, vol. 30, pp. 244-247, 1944.

[38] J. H. Weaver, C. G. Olson, and D. W. Lynch, "Optical properties of crystalline tungsten," Physical Review B, vol. 12, no. 4, pp. 1293-1297, 1975.

[39] L. V. Nomerovannya, M. M. Kirillova, and M. M. Noskov, "Optical properties of tungsten monocrystals," Soviet physics, JETP, vol. 33, p. 405, 1971.

[40] J. X. Zhang and L. C. Chen, "Pressure dependence of phonon dispersion in bcc tungsten," Chinese Journal of Physics, vol. 51, no. 2, pp. 359-367, 2013.

[41] A. D. B. Woods and S. H. Chen, "Lattice dynamics of molybdenum," Solid State Communications, vol. 2, no. 8, pp. 233-237, 1964.

[42] T. H. Geballe, "Superconductivity in the transition metals," Reviews of Modern Physics, vol. 36, no. 1, pp. 134-138, 1964.

[43] M. M. Kirillova and B. A. Charikov, "Study of the optical properties of transition metals," Optics and Spectroscopy, vol. 17, pp. 134-135, 1964.

[44] D. W. Lynch, C. G. Olson, and J. H. Weaver, "Optical properties of Ti, Zr, and Hf from 0.15 to $30 \mathrm{eV}$," Physical Review B, vol. 11, no. 10 , pp. $3617-3624,1975$.
[45] R. de Hoff, Thermodynamics in Materials Science, Taylor \& Francis, Boca Raton, Fla, USA, 2nd edition, 2006.

[46] A. A. Quong and A. Y. Liu, "First-principles calculations of the thermal expansion of metals," Physical Review B, vol. 56, no. 13, pp. 7767-7770, 1997.

[47] G. J. Ackland, X. Huang, and K. M. Rabe, "First-principles thermodynamics of transition metals: W, NiAl, and PdTi," Physical Review B: Condensed Matter and Materials Physics, vol. 68, no. 21, Article ID 214104, 2003.

[48] P. Giannozzi, S. de Gironcoli, P. Pavone, and S. Baroni, "Ab initio calculation of phonon dispersions in semiconductors," Physical Review B, vol. 43, no. 9, pp. 7231-7242, 1991.

[49] W. Windl, P. Pavone, K. Karch et al., "Second-order Raman spectra of diamond from ab initio phonon calculations," Physical Review B, vol. 48, no. 5, pp. 3164-3170, 1993.

[50] G. Onida, L. Reining, and A. Rubio, "Electronic excitations: density-functional versus many-body Green's-function approaches," Reviews of Modern Physics, vol. 74, no. 2, pp. 601$659,2002$. 

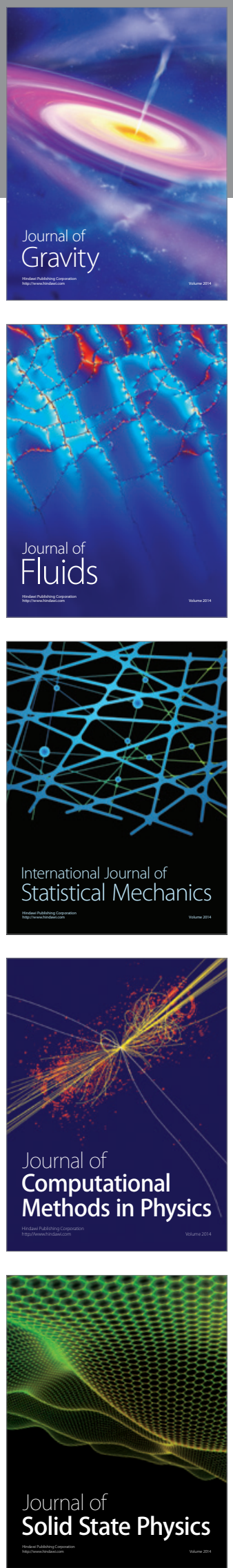

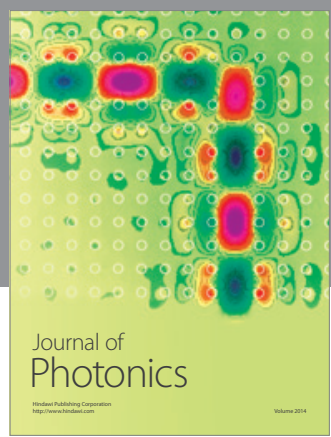

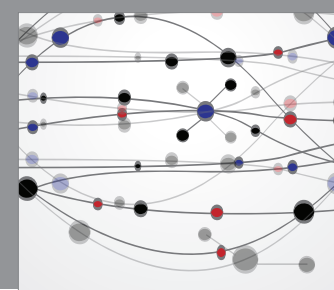

The Scientific World Journal

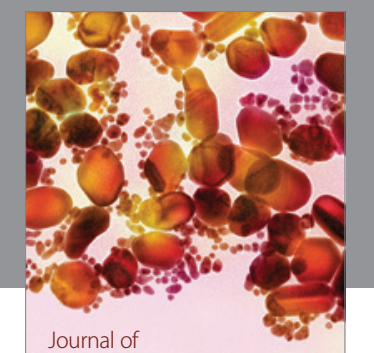

Soft Matter
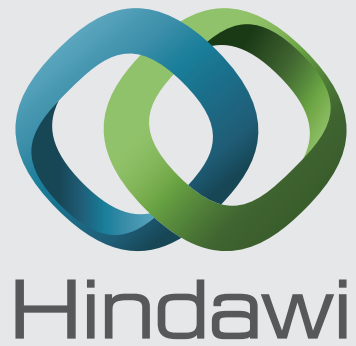

Submit your manuscripts at

http://www.hindawi.com
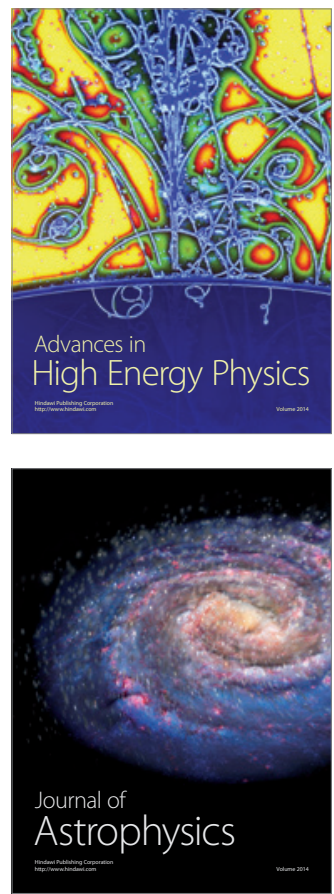
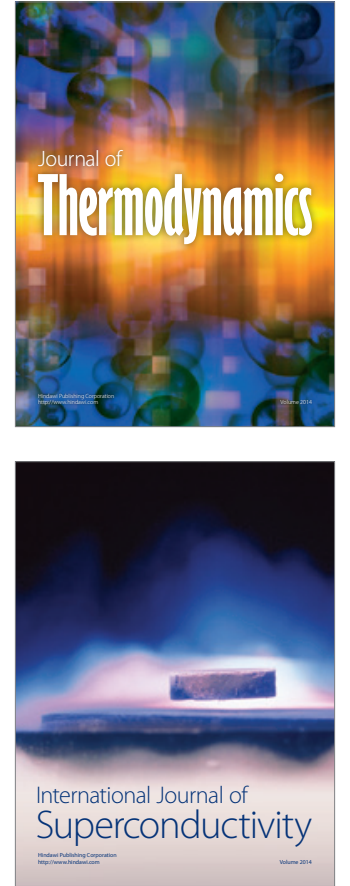
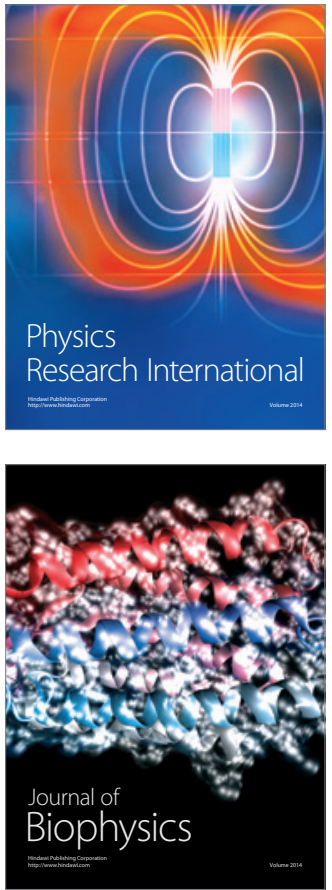
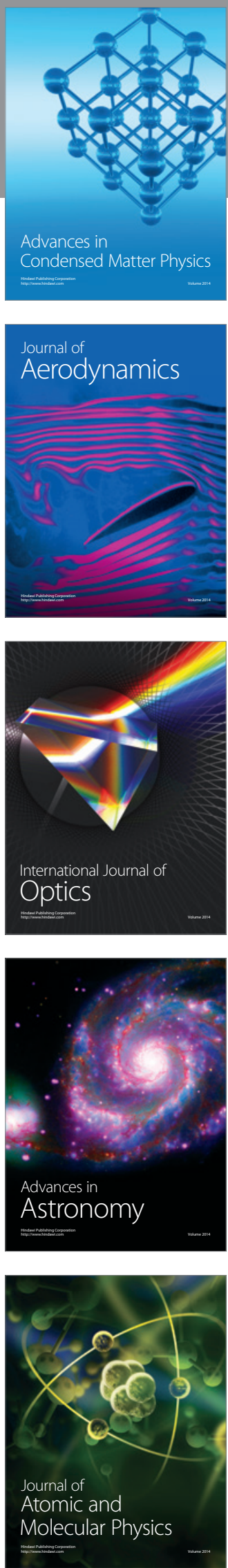